\title{
Morphological Evidence for the Sprouting of Inhibitory Commissural Fibers in Response to the Lesion of the Excitatory Entorhinal Input to the Rat Dentate Gyrus
}

\author{
T. Deller, ${ }^{1}$ M. Frotscher, ${ }^{1}$ and R. Nitsch ${ }^{2}$ \\ 'Institute of Anatomy, University of Freiburg, D-79001 Freiburg, Germany and 2Institute of Anatomy, Humboldt \\ University Berlin (Charité), D-10098 Berlin, Germany
}

\begin{abstract}
Recently a commissural fiber projection that terminates in the outer molecular layer of the fascia dentata was described in normal rats (Deller et al., 1995). In the present article, Phaseolus vulgaris leucoagglutinin (PHAL) tracing was used to analyze the contribution of this previously unknown projection to the commissural sprouting response after entorhinal cortex lesion. Rats 4-8 weeks after unilateral entorhinal lesion received a single PHAL deposit into the hilus of the fascia dentata contralateral to the lesion side. Unlesioned control animals received a similar PHAL deposit. The degree of axonal arborization and the bouton density per axon length were determined for individual PHAL-labeled commissural axons to the outer molecular layer of the dentate gyrus. A significant increase in both parameters was observed in the lesioned group. The PHALlabeled commissural fibers established symmetric synapses in the denervated outer molecular layer. Postembeddlng Immunocytochemistry Indlcated that some of these sprouting commissural fibers are GABAergic. Our findings provide morphological evidence for lamina-specific sprouting of an inhibitory commissural projection that normally terminates in the outer molecular layer. This suggests that inhibitory fibers participate in the replacement of the excitatory perforant pathway after entorhinal lesion.
\end{abstract}

[Key words: reactive synaptogenesis, neuronal specificity, neuronal plasticity, anterograde tracing, immunocytochemistry, hippocampal connections]

The rat fascia dentata has been used as a model to study the capacity of central neurons to reorganize following lesion (see Cotman and Nadler, 1978; Steward, 1991). This model is based on the observation that unilateral lesion of the entorhinal cortex (EC) results in the loss of approximately $85 \%$ of afferent fibers to the outer two-thirds of the dentate molecular layer (Matthews et al., 1976a; Steward and Vinsant, 1983), followed by sprouting of undamaged fibers that replace $60 \%$ to $80 \%$ of all lost synapses (Matthews et al., 1976b; Steward and Vinsant, 1983). This plastic response is said to involve several surviving afferent systems (review: Cotman and Nadler, 1978; Steward, 1991): (1) com-

\footnotetext{
Received May 8, 1995; revised June 5, 1995; accepted June 8, 1995.

We thank A. Schneider, R. Kovacs, M. Winter, and S. Kröber for technical assistance. This work was supported by the Deutsche Forschungsgemeinschaft (Fr 620/4-2, Ni 344/1-1, Ni 344/5-1, and Leibniz Program).

Correspondence should be addressed to Thomas Deller, M.D., Anatomisches Institut I, Postfach 111, 79001 Freiburg, Germany.

Copyright $\mathbb{C} 1995$ Society for Neuroscience $0270-6474 / 95 / 156868-11 \$ 05.00 / 0$
}

missural and associational fibers to the inner molecular layer expand their termination zone (e.g., Lynch et al., 1973, 1976; Zimmer et al., 1973; Goldowitz and Cotman, 1980; Lynch et al., 1982; West et al., 1984); (2) septohippocampal fibers, known to terminate throughout the molecular layer of the dentate gyrus, form a dense fiber plexus in the denervated zone (Lynch et al., 1972; Nadler et al., 1977; Nyakas et al., 1988); and (3) axons of the crossed temporo-dentate pathway participate in the reinnervation of the denervated septal portion of the hippocampal formation (Steward et al., 1974; Goldowitz et al., 1975; Deller et al., 1995b).

By using anterograde tracing with PHAL, which allows for the identification of projections at the level of single fibers, we have recently shown a commissural projection to the outer molecular layer of the fascia dentata in normal rats (Deller et al., 1995 ) in addition to the well-characterized projection to the inner molecular layer (Blackstad, 1956; Zimmer, 1971; Gottlieb and Cowan, 1973; Swanson et al., 1978; Laurberg, 1979; Berger et al., 1980; Swanson et al., 1981; Laurberg and Sorensen, 1981; Leranth and Frotscher, 1987; Deller et al., 1994). This raised the question whether the commissural projection that normally terminates in the outer molecular layer participates in the reorganization process after EC lesion. In continuation of our study on the normal distribution of the different commissural projections to the fascia dentata (Deller et al., 1995), we have therefore used anterograde tracing with PHAL in order to study the sprouting of commissural fibers to the fascia dentata following unilateral EC lesion. In addition, we employed immunocytochemistry in order to identify the transmitter of this previously unknown commissural projection to the outer molecular layer.

\section{Materials and Methods}

Animals. Fourty-eight adult male and female Sprague-Dawley rats (250-350 gm) housed under standard laboratory conditions were used in this study. Experimental animals were divided into two groups: control animals $(n=12)$, and animals with unilateral electrolytic lesions of the entorhinal cortex $(n=36)$ which survived between 4 and 8 weeks prior to iontophoretic application of PHAL into the dentate gyrus on the side contralateral to the entorhinal lesion. Four animals with unilateral entorhinal lesions were used for postembedding immunogold staining for GABA.

Surgical procedures. Stereotaxic surgery was performed under decp nembutal anesthesia ( $50 \mathrm{mg} / \mathrm{kg}$ body weight). A standard electrocoagulator was used to make a unilateral cut in the frontal and saggital plane between the entorhinal area and the hippocampus which resulted in the complete destruction of the ipsilateral entorhinal afferents to the fascia dentata (Figs. 1 $a, b, 2 a, b$ ). The following coordinates measured from the interaural line were used: frontal cut: AP $+1 ; \mathrm{L} 3$ to 7 ; V down to the base of the skull; saggital cut: AP +1 to $+4 ; \mathrm{L} 6.7 ; \mathrm{V}$ 
down to the base of the skull (Paxinos and Watson, 1986). Completeness of entorhinal lesions was verified macroscopically when the brains were sectioned on a Vibratome, and histochemically using the acetylcholinesterase (AChE) procedure described below (dense AChE staining in the outer molecular layer; see Lynch et al., 1972; Nadler et al., 1977).

PHAL iontophoresis. PHAL injection and immunostaining procedures have been described in detail elsewhere (Gerfen and Sawchenko, 1984). Surgical procedures were perfomed under deep nembutal anesthesia $(50 \mathrm{mg} / \mathrm{kg}$ body weight). Briefly, all animals $(n=48)$ received a iontophoretically delivered deposit of PHAL (2.5\% in $10 \mathrm{~mm}$ phosphate buffer $\mathrm{pH} 7.8$; Vector Laboratories, Burlingame, CA) into the hilar area of the dentate gyrus (coordinates from bregma: temporal: AP - 6.3; L 4.7; V 5.8; septal: AP: $-3.8 ; \mathrm{L}: 1.6 ; \mathrm{V}:-3.8$ ) via a stereotaxically positioned glass micropipette (tip diameter $15-30 \mu \mathrm{m}, 5 \mu \mathrm{A}$ positive current applied for $5 \mathrm{sec}$ followed by an off-period of $5 \mathrm{sec}$ for $20-30$ min). After a survival time of $10 \mathrm{~d}$ following the injection of the anterograde tracer, the rats were deeply anesthetized with an overdose of nembutal and were transcardially perfused with a fixative containing $4 \%$ paraformaldehyde, $0.08 \%$ glutaraldehyde, and $15 \%$ picric acid in $0.1 \mathrm{M}$ phosphate buffer (PB) ( $\mathrm{pH} 7.4)$. Those animals that were used for postembedding immunostaining for GABA were perfused with a fixative containing $2.5 \%$ paraformaldehyde, $1 \%$ glutaraldehyde, and $0.2 \%$ picric acid in $0.1 \mathrm{M}$ PB. Brains were removed and postfixed for $2 \mathrm{hr}$ in glutaraldehyde-free fixative. Horizontal or frontal sections (100 $\mu \mathrm{m}$ ) were cut with a Vibratome and washed in PB. Of those brains which had received a unilateral EC lesion, every fifth section was processed for AChE histochemistry to check for the completeness of the entorhinal lesion.

Immunocytochemistry was used to visualize PHAL-containing axons. Free-floating sections were incubated for $2 \mathrm{~d}$ at $4^{\circ} \mathrm{C}$ in biotinylated goat anti-PHAL (1:400, Vector Labs.), $1 \%$ normal horse serum and $0.1 \%$ $\mathrm{NaN}_{3}$ in $0.1 \mathrm{M}$ PB. For light microscopy, the antibody solution also contained $0.5 \%$ Triton $\mathrm{X}-100$. After rinsing in $\mathrm{PB}$, the sections were incubated in the intensified avidin-biotin-peroxidase complex (ABCElite, Vector Labs.) for $3 \mathrm{hr}$. Following three subsequent washes, the sections were immersed in a nickel/DAB solution $\left(0.05 \% 3,3^{\prime}\right.$ diaminobcnzidine, $0.02 \%$ nickel ammonium chloride, $0.024 \%$ cobalt chloride, $0.001 \% \mathrm{H}_{2} \mathrm{O}_{2}$, in $0.1 \mathrm{M} \mathrm{PB}, 5-10 \mathrm{~min}$ ) which resulted in a deep-blue labeling of PHAL containing fibers. Those animals that were used for postembedding iummunostaining for GABA were reacted with $\mathrm{DAB}$ alone $\left(0.05 \% 3,3^{\prime}\right.$ diaminobenzidine, $0.001 \% \mathrm{H}_{2} \mathrm{O}_{2}$, in $0.1 \mathrm{M} \mathrm{PB}, 5-10$ min). Sections for light microscopy were placed on gelatin-coated slides, dehydrated in ethanol, and mounted in Eukitt. The sections for electron microscopy were osmicated $\left(0.5 \% \mathrm{OsO}_{4}\right.$ in $\left.\mathrm{PB}, 30 \mathrm{~min}\right)$, dehydrated ( $70 \%$ ethanol containing $1 \%$ uranyl acetate), and embedded in Durcopan (ACM, Florida) between liquid release coated slides and coverslips. Selected sections from three control animals and three animals that survived a complete EC lesion for 4 weeks were reembedded in blocks, and ultrathin sections collected on single-slot Formvar-coated copper grids were contrasted with lead citrate and examined in a Zeiss electron microscope. Thin sections to be processed for postembedding GABA immunostaining were mounted on nickel grids.

Postembedding immunogold staining for GABA. The immunogold staining procedure followed that described by Somogyi and Hodgson (1985), using a commercially available antiserum against GABA (Sigma). The immunostaining was carried out on droplets of Milliporefiltered solutions in humid petri dishes. Briefly, immersion in $1 \%$ periodic acid $(10 \mathrm{~min}$ ) was followed by washing in several changes of double-distilled water. Thereafter, the grids were transferred through $2 \%$ sodium metaperiodate $(10 \mathrm{~min})$ and washed in several changes of double-distilled water, and in three changes of Tris-buffered saline (TBS, $\mathrm{pH}$ 7.4). After preincubation in $1 \%$ ovalbumin dissolved in TBS (30 min) the grids were incubated overnight in a rabbit anti-GABA antiserum (Sigma, 1:5000, in 1\% normal goat serum in TBS). Following rinsing in TBS and in $50 \mathrm{~mm}$ Tris buffer (pH 7.4) containing $1 \%$ bovine serum albumin and $0.5 \%$ Tween $20(10 \mathrm{~min})$, the grids were incubated in the secondary antibody (goat anti-rabbit IgG-coated colloidal gold. $15 \mathrm{~nm}$ ) for $2 \mathrm{hr}$ (diluted 1:10, darkness). Following rinsing in $2 \%$ glutaraldehyde $(10 \mathrm{~min})$ the grids were again washed in double-distilled water, and stained with uranyl acetate and lead citrate. In control experiments the primary GABA antibody was omitted. No immunogold labeling occurred under these conditions.

$A C h E$ histochemistry. After unilateral entorhinal lesion a dense band of AChE-positive fibers appears in the outer molecular layer of the dentate gyrus on the side of the lesion (Lynch et al., 1972; Nadler et al., 1977). Every fifth section of the brains with unilateral entorhinal lesions was processed for $\mathrm{AChE}$ histochemistry according to the method described by Mesulam et al. (1987). The appearance of the AChE band was used as a criterion for the completeness of the EC lesion (Fig. $2 a, b)$.

Statistical analysis. In order to analyze the total number of branches of individual commissural axons to the outer molecular layer, the axonal arbor as visualized in single sections was drawn with the aid of a camera lucida (magnification $1000 \times$ ). Fifteen axons from five animals 4-8 weeks after EC lesion and 15 axons from the control group were drawn (see Results). In these drawings, the order of each branch was determined, beginning with the main axon (order \#1). Branches of the main axon were assigned order \#2 and so forth. To find out the degree of collateralization of an axon, the total number of branches of a given branch order was determined. The numbers of branches per order were summed up, averaged over the total number of axons, and the standard error of the mean (SEM) was calculated (see Fig. $6 a$ ). In addition, the total number of branches per axon was summed up for the control and the EC lesioned group, and the two groups were tested against each other using the Wilcoxon/Mann-Whitney test (see Fig. $6 b$ ). Similarly, the number of boutons per axon was counted, expressed as boutons per $100 \mu \mathrm{m}$ axon length, and tested for significance (see Fig. $6 \mathrm{c}$ ). A bouton was defined as a localized swelling of the axon beyond the normal width of this axon. Finally, an alpha correction was made according to Bonferroni and Holm (Holm, 1979), to take multiple testing of the same material into account.

\section{Results}

\section{Control animals}

These animals received a single PHAL deposit into the hilus. In nine cases the PHAL deposit was correctly located in the dentate gyrus, and PHAL-labeled commissural fibers were found contralaterally. The injection sites covered major portions of the hilar area, occasionally included CA3 pyramidal neurons, and frequently granule cells. Injection sites varied in diameter between 50 and $300 \mu \mathrm{m}$ (see also Deller et al., 1995). On the contralateral side many commissural fibers participated in the formation of the well-known fiber plexus in the inner molecular layer. Commissural fibers to the outer molecular layer passed through this inner plexus without branching. These fibers arborized close to the hippocampal fissure and gave off short recurrent collaterals (Fig. 3a; Deller et al., 1995). Five animals showed axonal arbors of commissural fibers that could be traced from the main axon traversing the inner molecular layer to the terminal branches in the outer molecular layer. These commissural fibers were used in our statistical analysis.

\section{Animals with entorhinal lesions}

Twenty-six animals displayed a completc cntorhinal lesion or a complete cut through the perforant pathway as ascertained both visually and by using the presence of an AChE-positive band as a criterion (see Materials and Methods; Fig. 2a,b). In 18 animals with complete lesions, the PHAL deposit was 10cated in the hilus of the dentate gyrus and PHAL-labeled commissural fibers were found contralaterally. No differences were observed between animals 4 and 8 weeks after EC lesion. The PHAL-injection sites and the trajectory of commissural fibers to the outer molecular layer were similar to those seen in normal rats. In contrast, PHAL-labeled commissural fibers to the outer molecular layer were more abundant in lesioned animals when compared to controls (Fig. $2 c, d$ ). No detailed analysis of fibers contributing to the main (inner) plexus was performed in the present study, since changes in the inner plexus after $\mathrm{EC}$ lesion were the subject of previous investigations (e.g., Lynch et al., 1973; Zimmer, 1973; Goldowitz and Cotman, 1980). Commissural fibers directly heading to the outer molecular lay- 

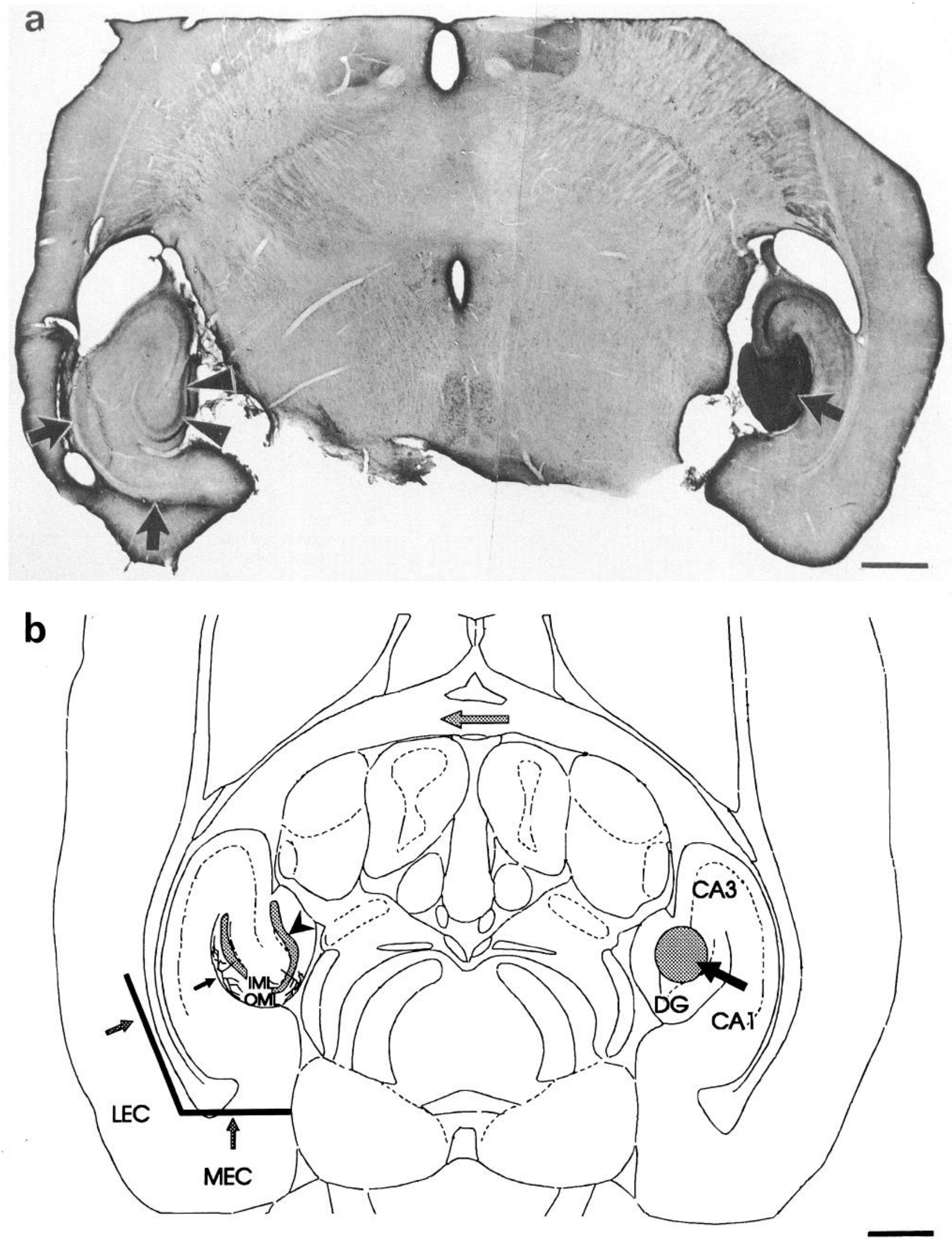

Figure 1. Demonstration of the methodological approach. Unilateral lesion of the entorhinal cortex (left-handed side) was followed by PHAL injection into the contralateral hilus to label sprouting commissural fibers. $a$, Horizontal section of a rat brain developed for PHAL immunocytochemistry. Note the site of the PHAL deposit (bold arrow on the right) and the commissural fiber projection on the side of the lesion (arrowheads) 
er were seen in all lesioned animals. Five animals displayed axonal arbors to the outer molecular layer that could be followed in their entirety from the main axon traversing the inner molecular layer to the terminal branches in the outer molecular layer (Fig. 3b). These animals were used for our statistical analysis. The axonal arborization and the bouton density of these fibers were analyzed and compared with the same parameters of control axons. Figures $3 a$ and $4 a$ illustrate a typical commissural fiber to the outer molecular layer in a control animal. In comparison, Figures $3 b$ and $4 b$ show a typical commissural fiber to the outer molecular layer after EC lesion. Note the increased number of axon branches and the high density of boutons of the commissural axon to the outer molecular layer after EC lesion as compared to the control axon. In addition, several boutons were very large, unlike any seen in control animals. Statistical analysis of a large number of axons confirmed that the total number of branches of a single commissural axon to the outer molecular layer was significantly greater after entorhinal lesion than in controls (Fig. 6a,b). Likewise, the bouton density was significantly greater than in controls (Fig. 6c). We hypothesize that collateral sprouting underlies the increase in the number of branches of commissural axons to the outer molecular layer seen in our material and that this axonal growth is followed by the formation of new synapses. Electron microscopic examination of these light microscopically observed boutons revealed that most if not all of these swellings represented synapses. Often en passant synapses were observed, and the contacts formed appeared to be symmetric (Fig. $5 a, b$ ). It should be pointed out, however, that the nature of the contact could not always be determined with certainty. In comparison to control animals, it was much easier to find PHAL-labeled boutons in the outer molecular layer in thin sections of animals that survived for 4-8 weeks after EC lesion.

To determine the neurotransmitter of the commissural fibers to the outer molecular layer, PHAL-labeled axons in the outer one-third of the molecular layer were serially sectioned and processed for postembedding GABA immunogold labeling. Axon terminals immunoreactive for PHAL were found to be immunoreactive for GABA and appeared to establish symmetrical synapses in the outer molecular layer (Fig. $5 c, d$ ). As one would expect, in the same material numerous GABA-immunoreactive terminals without PHAL-immunoreactivity were seen (Fig. 5e), demonstrating that colloidal gold particles did not attach selectively to PIIAL-labeled structures.

\section{Discussion}

The results of the present study can be summarized as follows: The commissural projection that normally terminates in the outer molecular layer shows sprouting within the entire denervated zone 4-8 weeks after unilateral EC lesion. Axon collateralization and bouton density of single fibers were significantly increased by more than twofold when compared to similar fibers of control animals. Postembedding immunocytochemistry indicates that these commissural fibers sprouting after EC lesion are GABAergic. Our data provide morphological evidence for laminaspecific sprouting of an inhibitory commissural projection that normally terminates in the denervated outer molecular layer. This suggests that inhibitory fibers participate in the replacement of the excitatory perforant-pathway after entorhinal lesion.

\section{Methodological considerations}

Earlier studies which analyzed the commissural projection after EC lesion were based on anterograde degeneration (e.g., Lynch et al., 1973, 1978; Zimmer et al., 1973), autoradiography (Goldowitz and Cotman, 1980), and anterograde HRP tracing (West, 1984) that do not depict individual sprouted fibers. Here we have used anterograde tracing with the highly sensitive and specific tracer PHAL (Gerfen and Sawchenko, 1984) that allows for the identification of projuctions at the single fiber level. This tracer has been employed previously to demonstrate sprouting of the septo-hippocampal fibers (Nyakas et al., 1988). As far as the commissural projection is concerned, we have recently shown a hitherto unknown commissural projection to the outer molecular layer of the fascia dentata in normal rats with the aid of this technique (Deller et al., 1995).

In the present study, we have traced individual PHAL-labeled commissural fibers in order to analyze the sprouting of the commissural projection to the outer molecular layer after EC lesion directly. This approach appeared advantageous, since neurons that take up the tracer are completely labeled, and their axonal arbor can thus be analyzed (Gerfen and Sawchenko, 1984). Variations in the size and location of PHAL-injections do only affect the number of labeled axons but not the labeling of individual fibers. I ikewise, shrinkage of the outer molecular layer (e.g., Matthews et al., 1976a; Steward and Vinsant, 1983) that could mimic an increase in fiber density in the denervated zone, does not affect the analysis of individual axons. Shrinkage alone could neither increase axon collateralization nor bouton density of single axons. Nevertheless, one might still argue that shrinkage has led to a smaller distance between boutons on the same axon branch which could mimic an increase in bouton density. Although this would imply an increased tortuosity of axons that we did not see in our material, we used our data and corrected bouton density for a $30 \%$ shrinkage. We still found significant ( $p<0.01$ ) changes between the control and the EC lesioned group which makes it very unlikely that the effects observed in the outer molecular layer are due to shrinkage.

\section{Lamina-specific sprouting of the commissural projection to the outer molecular layer}

The commissural projection to the outer molecular layer of the fascia dentata terminates in the same lamina as the entorhinal fibers that reach the fascia dentata via the perforant pathway. Our results show that this commissural projection sprouts exclusively within this layer after EC lesion and thus respects the laminar organization of the fascia dentata. This stands in marked contrast to earlier reports on the sprouting of the commissural

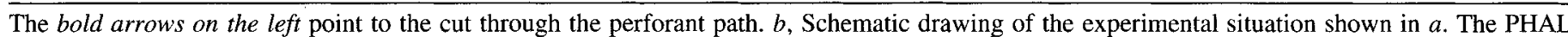

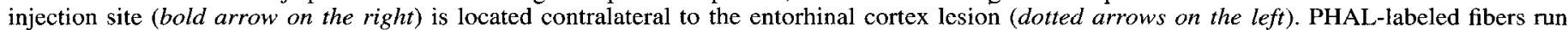

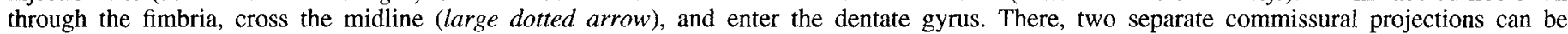

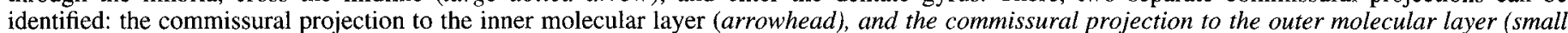

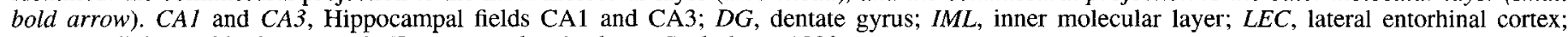
$M E C$, medial entorhinal cortex; $O M L$, outer molecular layer. Scale bars, $1000 \mu \mathrm{m}$. 

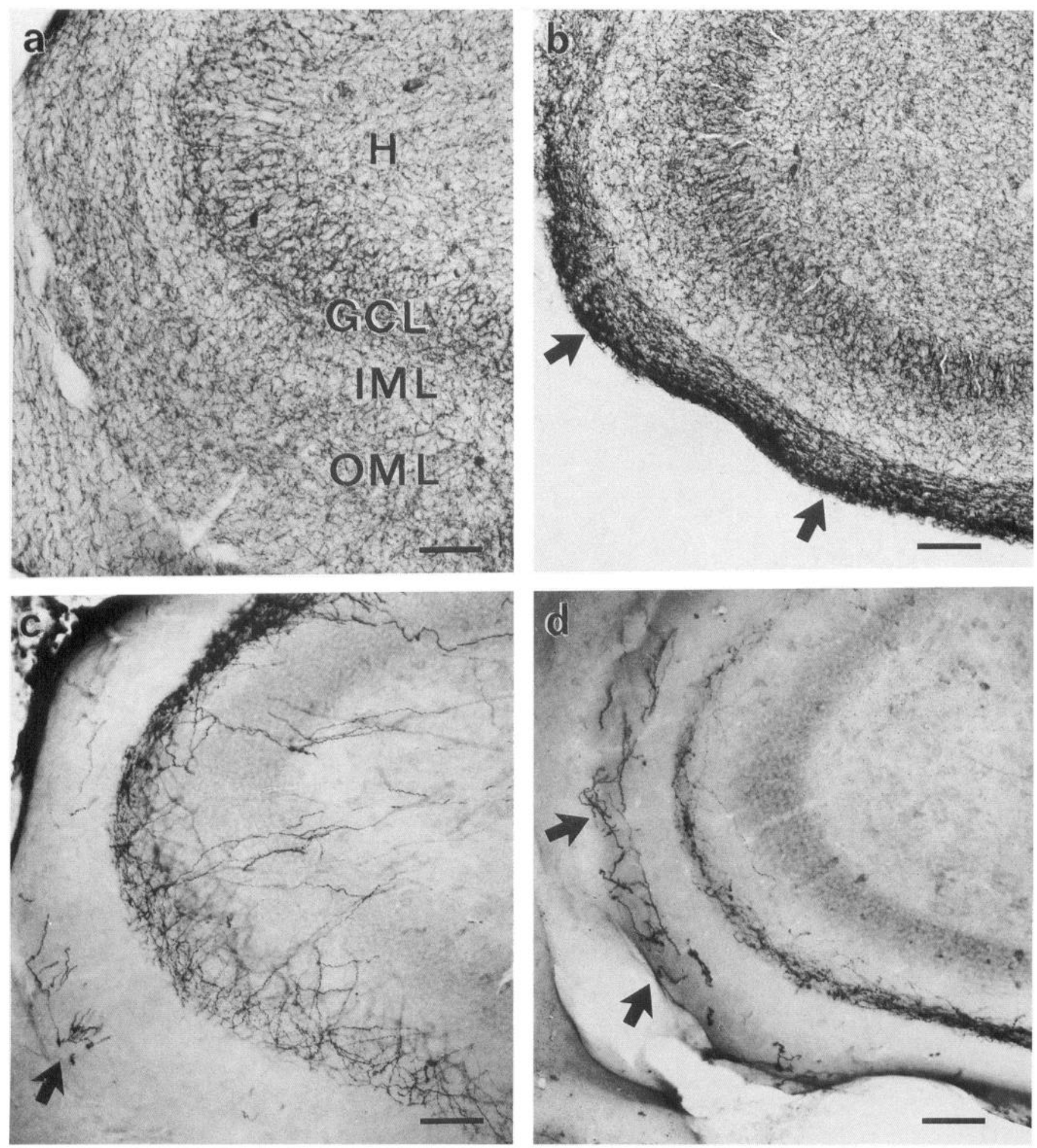

Figure 2. Sprouting in the outer molecular layer of the dentate gyrus after entorhinal lesion. $a$, AChE-stained section of the dentate gyrus in a control animal. $G C L$, Granule cell layer; $H$, hilus; $I M L$, inner molecular layer; $O M L$, outer molecular layer. $b$, AChE-stained section of the dentate gyrus 8 weeks after entorhinal cortex lesion. Note dense AChE-positive fiber plexus present in the outer molecular layer (arrows). $c$, Representative section of the dentate gyrus in an unlesioned control animal with a PHAL injection into the contralateral hilus. The sparse commissural projection to the outer molecular layer is indicated by an arrow. This section was not cut precisely perpendicular to the longitudinal axis of the hippocampus. Therefore, the width of the granule cell layer and the molecular layer appears relatively large. $d$, Representative section of the dentate gyrus of an animal eight weeks after entorhinal cortex lesion. In this section, the commissural projection to the outer molecular layer (arrows) has considerably increased in fiber density as compared to the control animal. Scale bars, $100 \mu \mathrm{m}$. 

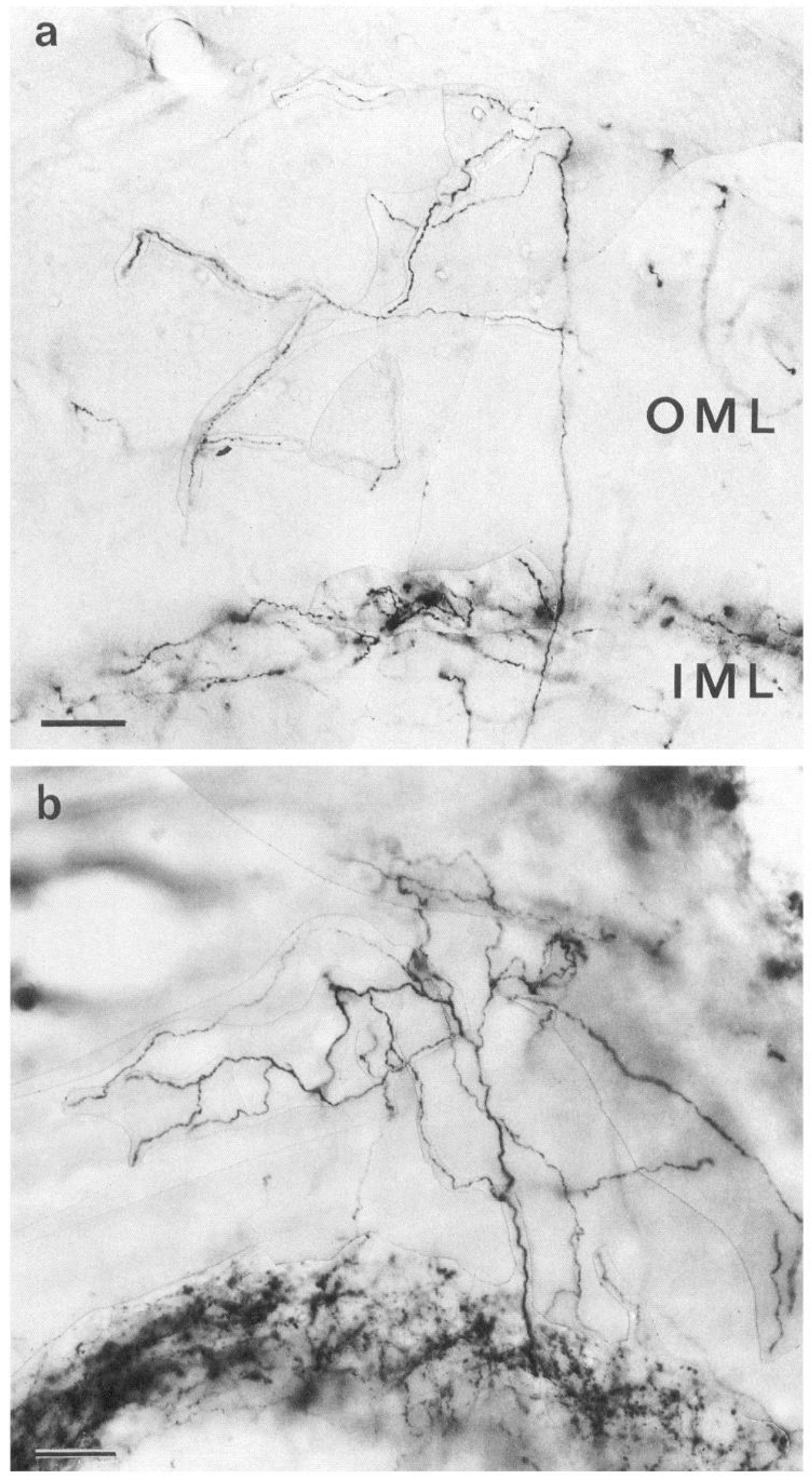

Figure 3. Photomontage of PHAL-labeled commissural axons to the outer molecular layer in a control $(a)$ and a lesioned animal $(b) . a$, Commissural axons to the outer molecular layer do not give off branches to the inner plexus, and the number of collaterals in the outer molecular layer is low in controls. A detailed camera lucida drawing of this axon is shown in Figure $4 a$. $I M L$, Inner molecular layer; $O M L$, outer molecular layer. $b$, Four weeks after entorhinal lesion the number of axonal collaterals has considerably increased in the outer molecular layer. Note that this axon does not branch in the inner molecular layer similar to the axon shown in $a$. A detailed camera lucida drawing of this axon is shown in Figure $4 b$. Scale bars, $30 \mu \mathrm{m}$. 
a

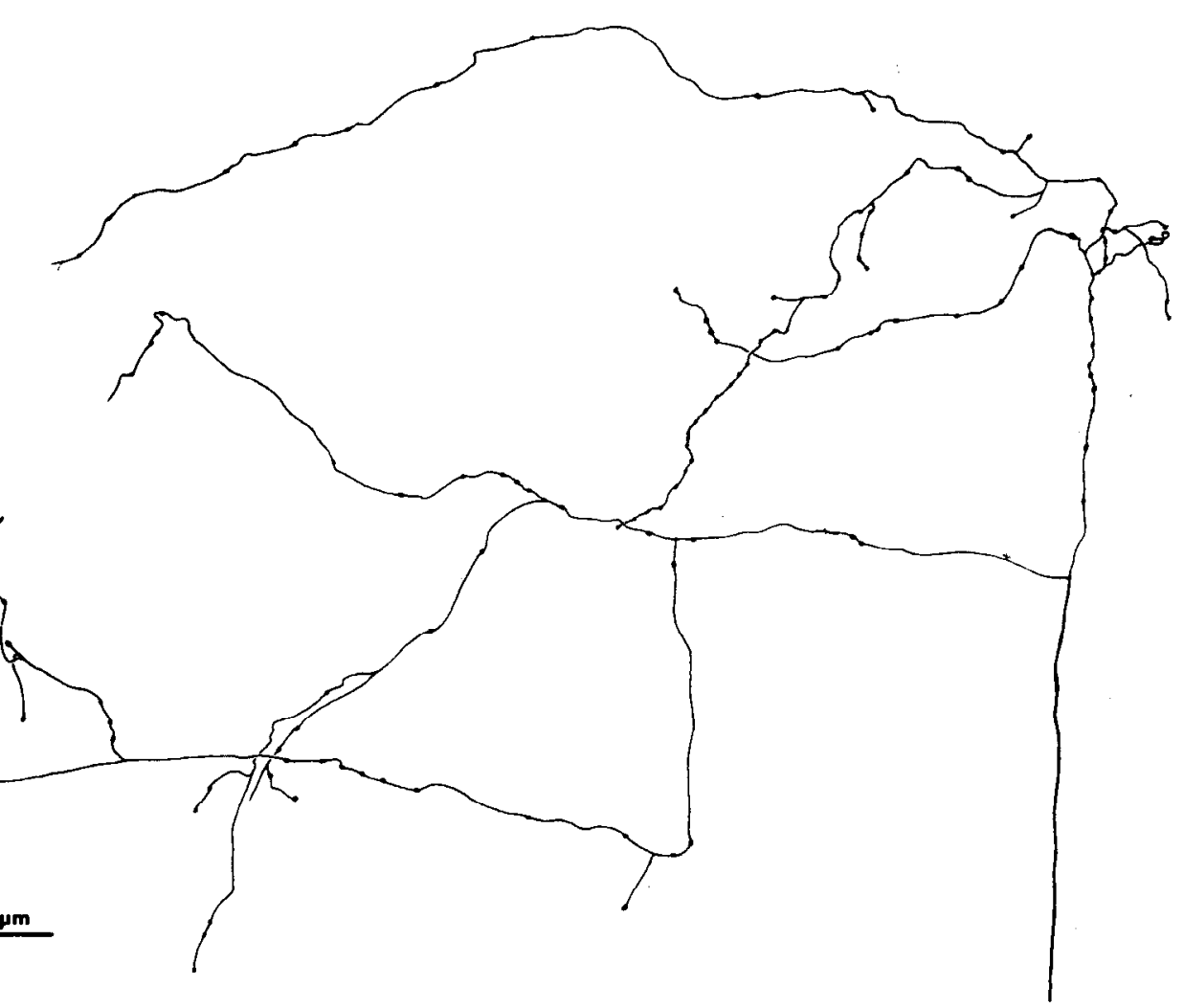

b

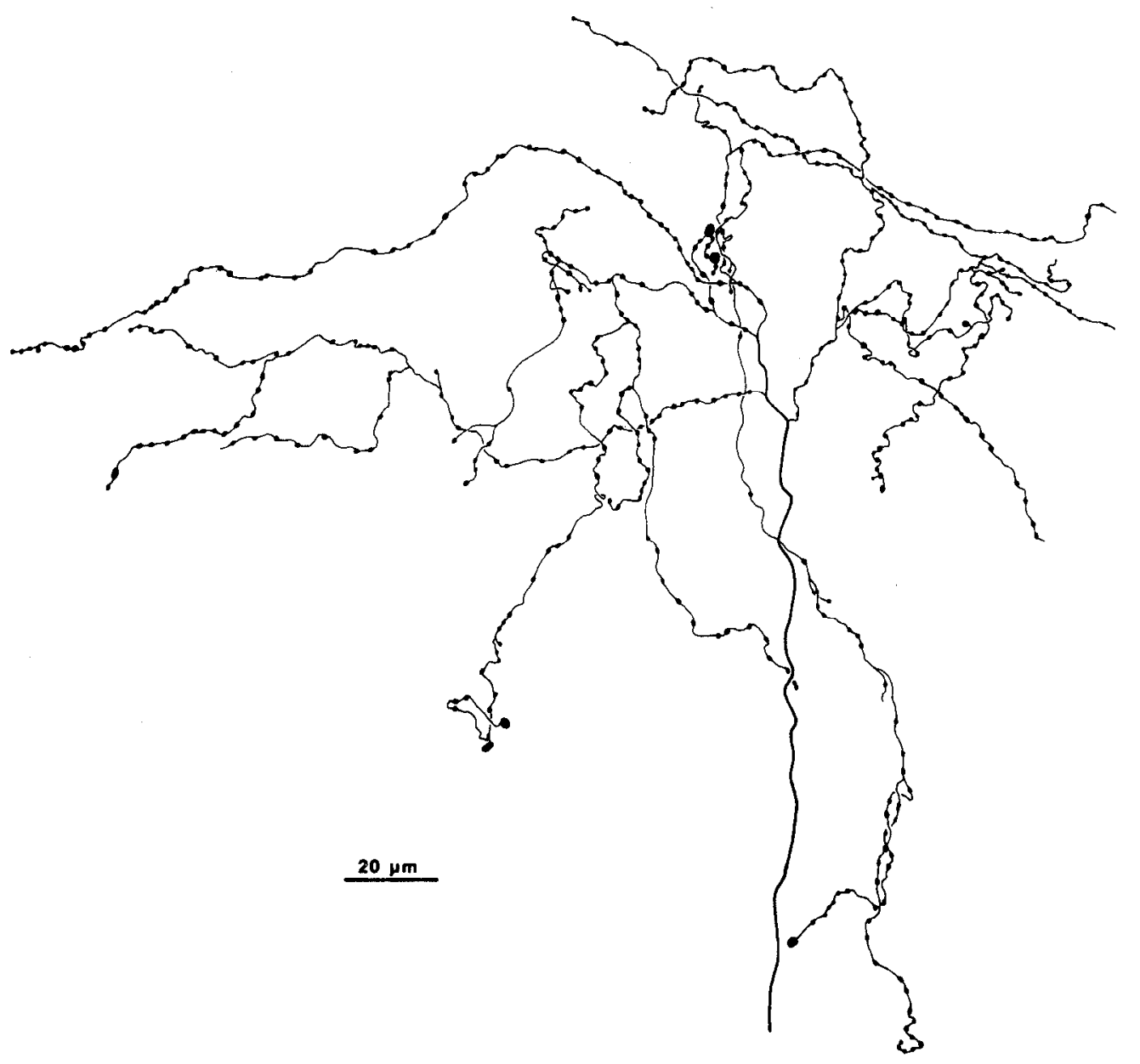


projection to the inner molecular layer, that is, a commissural projection that terminates in a zone that is not denervated by $\mathrm{EC}$ lesion. These fibers are said to leave their appropriate inner zone and sprout into the denervated outer molecular layer. This translaminar sprouting of the commissural projection to the inner molecular layer has becn inferred from studies that analyzed changes in the width of the termination zone of the commissural fibers (e.g., Lynch et al., 1973; Zimmer, 1973; West et al., 1975; Goldowitz and Cotman, 1980; Styren et al., 1994). These authors interpreted the expansion of the commissural/associational $(\mathrm{Cl}$ A) termination field as a sign for the invasion of C/A fibers into the denervated outer molecular layer. However, dendritic remodeling also occurs and first order stem dendrites of granule cells lengthen by $35 \mu \mathrm{m}$ (Caceres and Steward, 1983), which corresponds exactly to the increase in width reported for the $\mathrm{C} / \mathrm{A}$ fiber termination zone. Thus, the expansion of the inner molecular layer could also be regarded as resulting from intralaminar dendritic changes in combination with presynaptic remodelling which bring the synaptic density back to normal levels (Gall et al., 1979; see Steward, 1991, for review). Therefore, measurements of the width of the C/A fiber termination zone do not prove translaminar sprouting, since this would imply that $\mathrm{C} / \mathrm{A}$ fibers form new contacts at dendritic sites formerly occupied by entorhinal fibers. Similarly, commissural synapses found in the outer two-thirds of the molecular layer (see Lynch and Cotman, 1975) are no proof for translaminar sprouting, since these synapses could arise from the commissural projection that normally terminates in the outer molecular layer. Our data provide evidence for lamina-specific sprouting of the commissural projection that normally terminates in the outer molecular layer; translaminar sprouting of the inner commissural fiber plexus remains to be demonstrated.

\section{GABAergic sprouting after entorhinal lesion}

Although sprouting of GABAcrgic fibers in response to EC lesion has been suggested earlier based on an increase in glutamate-decarboxylase (GAD) activity (Nadler et al. 1974), GADimmunoreactivity (Goldowitz et al., 1982), and the postlesional desensitization of GABA receptors (Nadler et al., 1981), no direct evidence for sprouting of GABAergic fibers after EC lesion has yet been provided. In fact, it has been suggested that an increase in GAD activity could simply be due to shrinkage rather than sprouting (Storm-Mathiesen, 1974). Here we have demonstrated for the first time that GABAergic sprouting occurs following lesion of the excitatory perforant pathway. We have shown that the commissural projection to the outer molecular layer which sprouts following EC lesion forms symmetric synaptic contacts in the outer molecular layer. Moreover, these identified PHAL-labeled boutons were immunoreactive for the inhibitory neurotransmitter GABA. Thus, our data suggest a reorganization process where inhibitory fibers sprout in response to the loss of an excitatory fiber projection. This is of importance since the fact that lost fibers are replaced by remaining afferents might not signify a recovery of normal function.

\section{Heterolog sprouting versus homolog sprouting}

Another fiber system that has been shown to sprout following EC lesion is the crossed temporo-dentate pathway (CTD; Steward et al., 1973; Steward et al., 1976b; Deller et al., 1995b). This projection is anatomically homologous to the ipsilateral perforant pathway removed by EC lesion (Steward, 1976). Sprouting of the CTD has been implicated in the partial behavioral recovery occurring after EC lesion (Loesche and Steward, 1977; Steward, 1981; Ramirez and Stein, 1984; Schenk and Morris, 1985; Reeves and Smith, 1987) because the crossed entorhinal fibers exert a similar physiological effect (e.g., Harris et al., 1978; Reeves and Steward, 1986; Reeves and Steward, 1988) and replace the excitatory drive to the granule cells lost with the destruction of the ipsilateral perforant path. However, our findings provide direct morphological evidence for the sprouting of an anatomically and functionally heterolog GABAergic commissural fiber population occurring in parallel with the homolog CTD sprouting response. This could result in an increased granule cell inhibition after EC lesion that would counteract an increase in excitation gained by the sprouting of the CTD-pathway and interfere with the functional recovery after $\mathrm{EC}$ lesion.

\section{Long-lasting inhibition of dentate granule cells after EC lesion}

In fact, the capability of dentate granule cells to generate population spike responses upon orthodromic stimulation of their afferents is greatly reduced following EC lesion (Clusmann et al., 1994). This observation is indicative of an imbalance of excitation and inhibition in the denervated dentate gyrus even after long survival times. Moreover, the increase in granule cell inhibition cannot solely be explained with the expansion of the termination zone of the commissural projection to the inner molecular layer: an increase of inhibition of dentate granule cells was observed after stimulaton of sprouted fibers in the former perforant path termination zone that could not be detected upon stimulation of the inner molecular layer (Clusmann et al., 1994). One possible explanation for this increase in inhibition is an increased innervation (Nitsch et al., 1992) or efficacy (Nitsch and Frotscher, 1991; Clusmann et al., 1994) of inhibitory interneurons. The synaptic density on apical dendrites of GABAergic basket neurons in the denervated zone increases by about $20 \%$ in comparison with unlesioned controls (Nitsch et al., 1992). These inhibitory neurons are known to control the discharge of the granule cells, and overinnervation or increased efficacy of these inhibitory elements could effectively limit granule cell excitability. Our present results suggest a further possibility: sprouting of commissural GABAergic inhibitory fibers in response to EC lesion might result in increased granule cell inhibition. Sprouting of this inhibitory projection could also explain why commissural stimulation resulted in negative field potentials that almost reached the hippocampal fissure six weeks after lesion (West et al., 1975). Whether other inhibitory projections, such as the ipsilateral GABAergic associational projection to the outer molecular layer (see Amaral and Witter, 1995), also sprout

\section{$\leftarrow$}

Figure 4. Camera lucida drawings of commissural axons to the outer molecular layer in a control $(a)$ and a lesioned animal $(b)$. $a$, Commissural axon of a control rat (illustrated in Fig. $3 a$ ). Note low density of boutons in the course of the axon. Fifteen axons of this type, taken from five control and five lesioned animals, were analyzed for their collateralization and bouton density. $b$, Commissural axon to the outer molecular layer illustrated in Figure $3 b$ ( 4 weeks after EC lesion). Note increase in bouton density and occasional large size of boutons. 

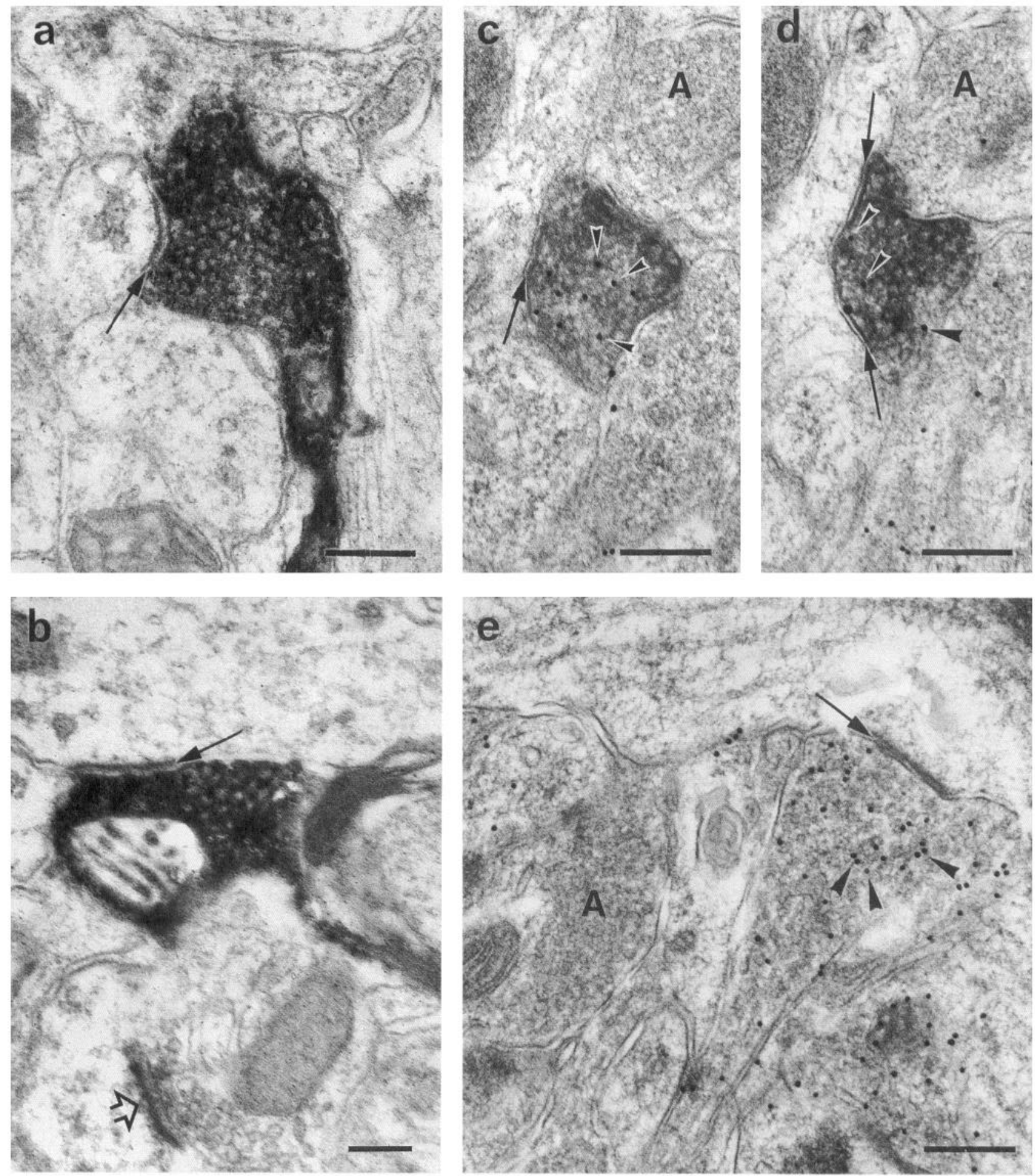

Figure 5. Electron micrographs of PHAL-labeled commissural fibers in the outer molecular layer of rats with EC lesions. $a$, Terminal forming symmetric synaptic contact (arrow). $b$, Terminal establishing symmetric synaptic contact with a dendritic shaft (arrow). The open arrow points to a neighboring unlabeled asymmetric synapse. $c$ and $d$, Serial sections of a commissural terminal double labeled for PHAL and GABA using a postembedding immunogold procedure. The arrows point to what appears to be symmetric membrane specializations. Arrowheads label some of the colloidal gold particles within the PHAL-labeled terminal. $A$, immunonegative axon terminal. $e$, PHAL-negative but GABA-positive bouton establishing symmetric synaptic contact (arrow). A neighboring axon terminal devoid of colloidal gold is indicated in the same section $(A)$. Scale bars: $a$ and $c-e, 0.25 \mu \mathrm{m} ; b 0.1 \mu \mathrm{m}$. 


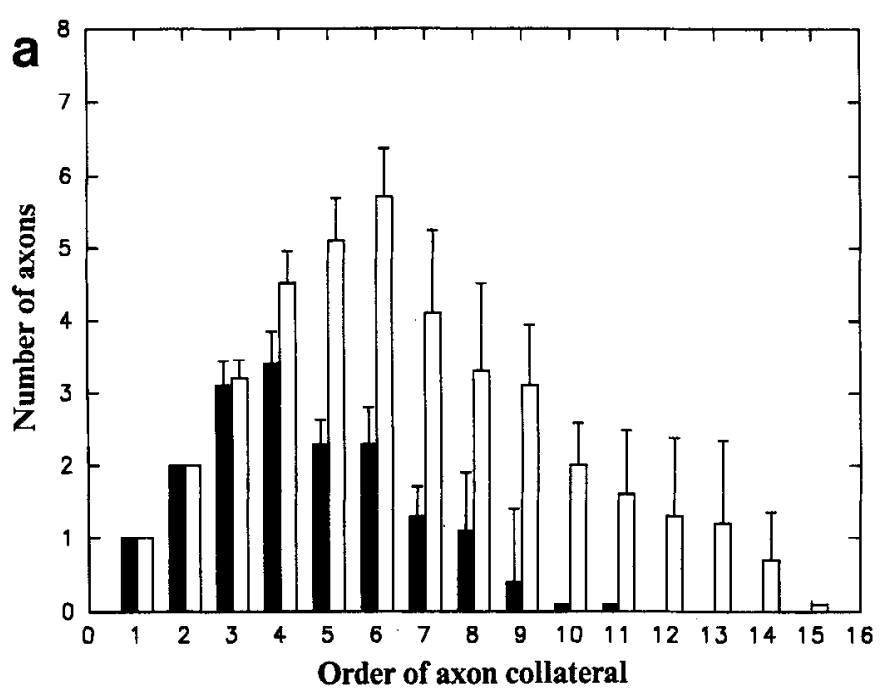

Controls

$\square$ Animals 4 - 8 weeks after entorhinal cortex lesion
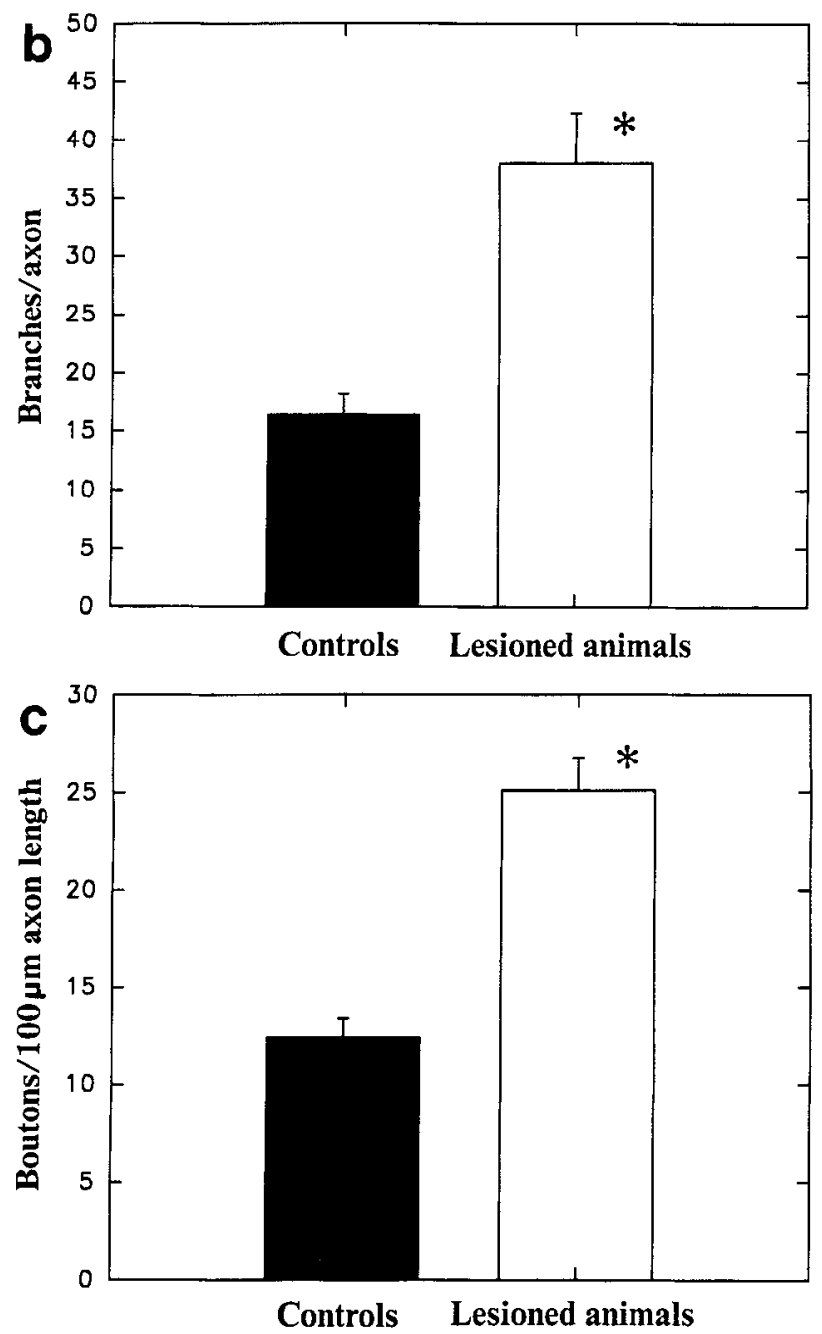

Figure 6. Quantitative analysis of the commissural projection to the outer molecular layer after entorhinal cortex lesion. $a$, Degree of arborization of single commissural axons to the outer molecular layer in controls and lesioned animals. The number of branches was larger in the EC lesioned group and the maximum number of branches shifted and contribute to the increased inhibition of the dentate gyrus after EC lesion remains to be elucidated.

\section{References}

Amaral D, Witter MP (1995) The hippocampal formation. In: The rat nervous system, 2d ed (Paxinos G, ed), pp 443-494. San Diego: Academic.

Berger TW, Semple-Rowland S, Basset J (1980) Hippocampal polymorph neurons are the cells of origin for ipsilateral association and commissural afferents to the dentate gyrus. Brain Res 215:329-336.

Blackstad TW (1956) Commissural connections of the hippocampal region of the rat, with special reference to their mode of termination. J Comp Neurol 105:417-537.

Caceres A, Steward O (1983) Dendritic reorganization in the denervated dentate gyrus of the rat following entorhinal cortical lesions: a Golgi and electron microscopic analysis. J Comp Neurol 214:387403.

Clusmann H, Nitsch R, Heinemann U (1994) Long lasting functional alterations in the rat dentate gyrus following EC lesion: a current source density analysis. Neuroscience 61:805-815.

Cotman CW, Nadler JV (1978) Reactive synaptogenesis in the hippocampus. In: Neuronal plasticity (Cotman CW, ed), pp 227-271. New York: Raven.

Deller T, Nitsch R, Frotscher M (1994) Associational and commissural afferents of parvalbumin-immunoreactive neurons in the rat hippocampus: a combined immunocytochemical and PHA-L study. J Comp Neurol 350:612-622.

Deller T, Nitsch R, Frotscher M (1995) Phaseolus vulgaris leucoagglutinin (PHAL) tracing of commissural fibers to the rat dentate gyrus: evidence for a previously unknown commissural projection to the outer molecular layer. J Comp Neurol 352:55-68.

Gall C, McWilliams R, Lynch G (1979) The effect of collateral sprouting on the density of innervation of normal target sites: implications for theories on the regulation of the size of the developing synaptic domains. Brain Res 175:37-47.

Gerfen CR, Sawchenko PE (1984) An anterograde neuroanatomical tracing method that shows the detailed morphology of neurons, their axons and terminals: immunohistochemical localization of an axonally transported plant lectin, Phaseolus vulgaris leucoagglutinin (PHAL). Brain Res 290:219-238.

Goldowitz D, Cotman CW (1980) Axonal transport and axon sprouting in the adult rat dentate gyrus: an autoradiographic study. Neuroscience 5:2163-2174.

Goldowitz D, White WF, Steward O, Cotman CW, Lynch G (1975) Anatomical evidence for a projection from the entorhinal cortex to the contralateral dentate gyrus of the rat. Exp Neurol 47:433-441.

Goldowitz D, Vincent SR, Wu J-Y, Hokfelt T (1982) Immunohistochemical demonstration of plasticity in GABA neurons of the adult rat dentate gyrus. Brain Res 238:413-420.

Gottlieb DI, Cowan WM (1973) Autoradiographic studies of the commissural and ipsilateral association connections of the hippocampus and dentate gyrus of the rat. I. The commissural connections. J Comp Neurol 149:393-422.

Harris EW, Lasher SS, Steward O (1978) Habituation like decrements in transmission along the normal and lesion-induced temporodentate pathways in the rat. Brain Res 151:623-631.

Holm S (1979) A simple sequentially rejective multiple test procedure. Scand J Stat 6:65-70.

Laurberg S (1979) Commissural and intrinsic connections of the rat hippocampus. J Comp Neurol 184:685-708.

Laurberg S, Sorensen KE (1981) Associational and commissural collaterals of neurons in the hippocampal formation (hilus fasciae dentatae and subfield CA3). Brain Res 212:287-300.

Leranth C, Frotscher M (1987) Cholinergic innervation of hippocampal

$\leftarrow$

to higher orders in the lesioned animals as compared to controls. $b$, Total number of branches of commissural axons to the outer molecular laycr. *, Significant difference between controls and lesioned animals $(p<0.001$; Wilcoxon/Mann-Whitney test). $c$, Bouton density of commissural axons to the outer molecular layer. *, Significant difference between controls and lesioned animals $(p<0.001$; Wilcoxon/MannWhitney test). 
GAD- and somatostatin-immunoreactive commissural neurons. J Comp Neurol 261:33-47.

Loesche J, Steward O (1977) Behavioral correlates of denervation and reinnervation of the hippocampal formation of the rat: recovery of alternation performance following unilateral entorhinal cortex lesions. Brain Res Bull 2:31-39.

I ynch G, Cotman CW (1975) The hippocampus as a model for studying anatomical plasticity in the adult brain. In: The hippocampus, Vol 1 (Isaacson RL, Pribram KH, eds), pp 123-154. New York: Plenum.

Lynch G, Matthews DA, Mosko S, Parks T, Cotman CW (1972) Induced acetylcholinesterase-rich layer in rat dentate gyrus following entorhinal lesions. Brain Res 42:311-318.

Lynch G, Stanfield B, Cotman CW (1973) Developmental differences in postlesion axonal growth in the hippocampus. Brain Res 59:155168.

Lynch G, Gall C, Rose G, Cotman CW (1976) Changes in the distribution of the dentate gyrus associational system following unilateral or bilateral entorhinal lesion in the adult rat. Brain Res 110:57-71.

Lynch G, McWilliam R, Gall C (1982) The effects of successive lesions on the time course of the sprouting response in the hippocampus of the rat. Brain Res 240:154-157.

Matthews DA, Cotman CW, Lynch G (1976a) An electron microscopic study of lesion-induced synaptogenesis in the dentate gyrus of the adult rat. I. Magnitude and time course of degeneration. Brain Res 115:1-21.

Matthews DA, Cotman CW, Lynch G (1976b) An electron microscopic study of lesion-induced synaptogenesis in the dentate gyrus of the adult rat. II. Reappearance of morphologically normal synaptic contacts. Brain Res 115:23-41.

Mesulam M-M, Geula C, Morán MA (1987) Anatomy of cholinesterase inhibition in Alzheimer's disease: effect of physostigmine and tetrahydroaminoacridine on plaques and tangles. Ann Neurol 22:683691

Nadler JV (1981) Desensitization-like changes in GABA-receptor binding of rat fascia dentata after entorhinal lesion. Neurosci Lett 26: 275-281.

Nadler JV, Cotman CW, Lynch G (1974) Biochemical plasticity of short-axon interneurons: increased glutamate decarboxylase activity in the denervated area of the rat dentate gyrus following entorhinal lesion. Brain Res 45:403-413.

Nadler JV, Cotman CW, Lynch G (1977) Histochemical evidence of altered development of cholinergic fibers in the rat dentate gyrus following lesions. I. Tinte course after complete unilateral entorhinal lesion at various ages. J Comp Neurol 171:561-588.

Nitsch R, Frotscher M (1991) Maintenance of peripheral dendrites of GABAergic neurons requires specific input. Brain Res 554:304-307.

Nitsch R, Bader S, Frotscher M (1992) Reorganization of input synapses of parvalbumin-containing neurons in the rat fascia dentata following entorhinal lesion. Neurosci Lett 135:33-36.

Nyakas C, Luiten PGM, Balkan B, Spencer DG Jr (1988) Changes in septohippocampal projections after lateral entorhinal or combincd cntorhinal-raphé lesions as studied by anterograde tracing methods. Brain Res Bull 21:285-293.

Paxinos G, Watson C (1986) Atlas of the rat brain in stereotaxic coordinates. Sidney: Academic.

Ramirez JJ, Stein DG (1984) Sparing and recovery of spatial alternation performance after entorhinal cortex lesions in rats. Behav Brain Res 13:53-61.

Reeves TM, Smith DC (1987) Reinnervation of the dentate gyrus and recovery of alternation behavior following entorhinal cortex lesions. Behav Neurosci 101:179-186.

Reeves TM, Steward O (1986) Emergence of the capacity for LTP during reinnervation of the dentate gyrus: evidence that abnormally shaped spines can mediate LTP. Exp Brain Res 65:167-175.

Reeves TM, Steward O (1988) Changes in the firing properties of neurons in the dentate gyrus with denervation and reinnervation: implications for behavioral recovery. Exp Neurol 102:37-49.

Schenk F, Morris RGM (1985) Dissociation between components of spatial memory in rats after recovery from the effects of retrohippocampal lesions. Exp Brain Res 58:11-28.

Somogyi P, Hodgson AJ (1985) Antisera to gamma-aminobutyric acid. III. Demonstration of GARA in Golgi-impregnated neurons and in conventional electron microscopic sections of cat striate cortex. J Histochem Cytochem 33:249-257.

Steward O (1976) Reinnervation of the dentate gyrus by homologous afferents following entorhinal cortex lesion in adult rats. Science 194: $426-428$.

Steward O (1981) Asscssing the functional significance of lesion-induced neuronal plasticity. Int Rev Neurobiol 23:197-254.

Steward O (1991) Synapse replacement on cortical neurons following denervation. Cereb Cortex 9:81-132.

Steward O, Vinsant SL (1983) The process of reinnervation in the dentate gyrus of the adult rat: a quantitative electron microscopic analysis of terminal proliferation and reactive synaptogenesis. J Comp Neurol 214:370-386.

Steward O, Cotman CW, Lynch G (1973) Re-establishment of electrophysiologically functional entorhinal cortical input to the dentate gyrus denervated by ipsilateral entorhinal lesions: innervation by the contralateral entorhinal cortex. Exp Brain Res 18:396-414.

Steward O, Cotman CW, Lynch G (1974) Growth of a new fiber projection in the brain of adult rats: reinnervation of the dentate gyrus by the contralateral entorhinal cortex following ipsilateral entorhinal lesion. Exp Brain Res 20:45-66.

Steward O, Cotman CW, Lynch GS (1976) A quantitative autoradiographic and electrophysiological study of the reinnervation of the dentate gyrus by the contralateral entorhinal cortex following ipsilateral entorhinal lesions. Brain Res 114:181-200.

Storm-Mathisen J (1974) Choline acetyltransferase and acetylcholinesterase in fascia dentata following lesion of the entorhinal afferents. Brain Res 80:181-197.

Swanson LW, Wyss JM, Cowan WM (1978) An autoradiographic study of the organization of intrahippocampal association pathways in the rat. J Comp Neurol 181:681-716.

Styren SE, DeKosky ST, Miller PD, Lagenaur CF (1994) L1 expression marks translaminar axon sprouting in rat dentate gyrus following entorhinal cortex lesion. Soc Neurosci Abstr 531.2.

Swanson LW, Sawchenko PE, Cowan WM (1981) Evidence for collateral projections by neurons in Ammon's horn, the dentate gyrus, and the subiculum-a multiple retrograde labeling study in the rat. $\mathbf{J}$ Neurosci 1:548-559.

West JR (1984) Age-dependent sprouting in the dentate gyrus demonstrated with anterograde HRP. Brain Res Bull 12:323-330.

West JR, Deadwyler S, Cotman WM, Lynch G (1975) Time-dependent changes in commissural field potentials in the dentate gyrus following lesions of the entorhinal cortex in adult rats. Brain Res 97:215-233.

Zimmer J (1971) Ipsilateral afferents to the commissural zone of the fascia dentata, demonstrated in decommissurated rats by silver impregnation. J Comp Neurol 142:393--416.

Zimmer J (1973) Extended commissural and ipsilateral projections in postnatally de-entorhinated hippocampus and fascia dentata demonstrated in rats by silver impregnation. Brain Res 64:293-311. 